\title{
AN EXAMINATION OF SOME CUT SETS OF SPACE*
}

\author{
BY C. H. HARRY
}

PART I

The purpose of this paper is to examine some pairs of points which are cut sets of a locally connected, locally compact, separable, and connected metric space $S$ which has no single cut point. Under such an hypothesis the following statement will be proved.

If $L$ is the set of all points ( $x$ ) such that $x$ together with some point $y_{x}$ separates two fixed points $a$ and $b$ of the space $S$, then $L+a+b$ is closed and compact. $\dagger$

By the pair $(x, y)$ separating $a$ and $b$ is meant that there exists at least one separation $S_{a}+S_{b}=S-x-y$ such that no point of $S_{a}$ is a point or limit point of $S_{b}$ and no point of $S_{b}$ is a limit point of $S_{a}$, where $a \subset S_{a}$ and $b \subset S_{b}$.

Two properties of $S$ used in the proof are the following:

I. Between $a$ and $b$ there exists at least one pair of $\operatorname{arcs} T_{x}$ and $T_{y}$ having just their end points $a$ and $b$ in common. $\ddagger$

II. If $X$ is any closed set, every component of $S-X$ is an arcwise connected open set with at least one limit point in $X . \S$

Properties of simple arcs which are used are the following:

III. If $x$ is any point of an arc $a b$, then $a b$ may be written as the sum of two arcs $a x$ and $x b$ having just $x$ in common.

IV. The points of an arc $a b$ may be ordered. If it is assumed that $a$ precedes $b, a \propto b$, the ordering gives the following relations:

* Presented to the Society, September 9, 1931.

$\dagger$ This result is analogous to the theorem of G. T. Whyburn, this Bulletin, vol. 33 (1927), p. 685, to the effect that if, in any locally connected and metric continuum $S, K$ is the set of all points separating two fixed points $a$ and $b$, then $K+a+b$ is closed and compact. See also R. L. Wilder, this Bulletin, vol. 34 (1928), p. 649.

$\ddagger$ See G. T. Whyburn, Proceedings of the National Academy of Sciences, vol. 13 (1927), pp. 31-38; and W. L. Ayres, American Journal of Mathematics, vol. 51 (1929), pp. 577-594. For a short proof of this theorem see G. T. Whyburn, this Bulletin, vol. 37 (1931), p. 429.

$\S$ R. L. Moore, Mathematische Zeitschrift, vol. 15 (1922). 
A point $x$ precedes a point $y, x \propto y$, if and only if $x \subset a y$ and $y \subset x b$, where $a b$ is written first as the sum of the arcs $a y$ and $y b$ and again as the sum $a x+x b$; if $x \propto y$, then $y$ does not precede $x$; if $x \propto y \propto z$, then $x \propto z$.

$\mathrm{V}$. If $K$ is any closed set and $a b$ any arc, the product $K \cdot a b$ has a last point on $a b$.

VI. If $\sum_{1}^{\infty} x_{i}$ is any monotonic plus* set of points on an arc $a b$ with limit point $p$ and $z$ is any point of the subarc $a p=a z+z p$ of $a b$, then $z p$ contains all but a finite number of the points $\sum_{1}^{\infty} x_{i}$.

The next lemma is of importance in fixing the pairs $(x, y)$.

LEMma. If $T_{x}$ and $T_{y}$ are any two arcs from $a$ to $b$ having just their end points $a$ and $b$ in common and $(x, y)$ is any pair of points separating $a$ and $b$, then $x$ is contained in one arc and $y$ in the other.

Proof. The assumption that one of the points is not contained in one of the arcs and the other point contained in the remaining arc easily leads to a contradiction, for then one of the arcs, say $T_{x}$, would contain neither $x$ nor $y$. Thus, $T_{x} \subset S-x-y$, which is impossible since the pair $(x, y)$ separates $a$ and $b$ while $T_{x}$ is a connected set containing both $a$ and $b . \dagger$

Since a simple arc is a compact set of points, the proof that $L$ is compact results immediately from the fact that $L \subset T_{x}+T_{y}$. Also, by choosing the order on $T_{x}$ and $T_{y}$ such that $a \propto b$ on both, a partial ordering of $L+a+b$ is established, e.g., a subset $Q$ of points $x$ of $L$ is said to be monotonic if it is monotonic with respect to the order of $T_{x}$. As the point $y$ also belongs to $L$ the $\operatorname{arcs}$ $T_{x}$ and $T_{y}$ form a division of $L$ into two parts $H_{x}=T_{x} \cdot L$ and $H_{y}=T_{y} \cdot L$. For the proof that $L+a+b$ is closed it will be assumed that a limit point $p$ of $L$ does not belong to $L+a+b$ and shown that this leads to a contradiction. Without loss it may be supposed that $p$ is a limit point of a monotonic plus set of points $\sum_{1}^{\infty} x_{i}$ of $H_{x}$. Two main cases then arise.

* The collection $\sum_{1}^{\infty} x_{i}$ is said to be monotonic plus if $x_{i} \propto x_{i+1}$ for each $i$. The collection is said to be monotonic minus provided $x_{i+1} \propto x_{i}$ for each $i$.

$\dagger$ From now on it will be assumed that one pair of the arcs $T_{x}$ and $T_{y}$ has been fixed and that the points $(x, y)$ have been so named that $x \subset T_{x}$ and $y \subset T_{y}$. 
CASE I. The corresponding set $\sum_{1}^{\infty} y_{i}$ of points $y_{i}$, which together with $x_{i}$ separate $a$ and $b$, consists of a finite number of distinct points.

If this be true, then an infinite number of the points $\sum_{1}^{\infty} x_{i}$ must be paired with one of the points of $\sum_{1}^{\infty} y_{i}$. Suppose that the pairs $\left(x_{n_{i}}, y_{k}\right)$, where $i=1,2,3, \cdots$, separate $a$ and $b$ and the $x_{n_{i}}$ 's are so labeled as to be monotonic. Now the pair $\left(p, y_{k}\right)$ does not separate $a$ and $b$ for $p$ is not a point of $L+a+b$. Hence, if $C$ is the component of $S-p-y_{k}$ containing $a$, then $C$ contains $b$. But, from Property II, a simple arc $T$, contained in $C$, exists from $a$ to $b$. Writing $T_{x}=a p+p b$ and using Property V, we see that the $\operatorname{arc} T$ has a last point $u$ on $a p$. Since $u \neq p$ the subarc $u p$ of $a p$ contains all but a finite number of the points of $\sum_{i=1}^{\infty} x_{n_{i}}$, Property VI. Thus, some $i$ exists such that $x_{n_{i}} \subset u p-u$. However, this is impossible for then $T$ would be a connected set containing both $a$ and $b$ and lying within $S-x_{n_{i}}-y_{k}$.

Since Case I leads to a contradiction there is left Case II.

CASE II. $\sum_{1}^{\infty} y_{i}$ consists of an infinite number of distinct points.

By choosing the $x_{i}$ 's so that the corresponding $y_{i}$ 's are monotonic on $T_{y}$, Case II may be divided into four parts:

A. The $y_{i}$ 's are monotonic plus with limit point $q \neq b$.

B. The $y_{i}$ 's are monotonic minus with limit point $q \neq a$.

C. The $y_{i}$ 's are monotonic plus with limit point $q=b$.

D. The $y_{i}$ 's are monotonic minus with limit point $q=a$.

Case II A. Exactly as before, the component $C$ of $S-p-q$ containing $b$ contains $a$ since $p$ is not a point of $L+a+b$. Also, an $\operatorname{arc} T$ from $a$ to $b$ exists such that $T \subset C$. Writing $T_{x}=a p+p b$ and $T_{y}=a q+q b$, then, just as in Case I, we see that the arc $T$ has a last point $u$ on $a p$ and a last point $v$ on $a q$. Likewise, from Property VI, the subarc $u p$ contains all but a finite number of the points $\sum_{1}^{\infty} x_{i}$ and the subarc $v q$ contains all but a finite number of the points $\sum_{1}^{\infty} y_{i}$. That is to say, there exists a number $K$ such that $T \subset S-x_{i}-y_{i}$ if $i>K$. But this is impossible since $T$ is a connected set containing both $a$ and $b$.

CASE II B. With exactly similar reasoning to that of Case II A it may be shown that this case again leads to a contradiction. 
There remain Cases II C and D, the latter of which will be treated next.*

CASE II D. From the fact that an arc minus its end point is a connected set it follows that $a x_{1}-x_{1} \subset S_{a_{i}}$ for every $i$, where $T_{x}=a x_{1}+x_{1} b$ and $S-x_{i}-y_{i}=S_{a_{i}}+S_{b_{i}}$, a separation of $S-x_{i}-y_{i}$ containing $a$ and $b$ respectively. Thus, if $z$ is a point of $a x_{1}-x_{1}$ $-a$ the pairs $\left(x_{i}, y_{i}\right)$ separate $z$ and $b$ as well as $a$ and $b$. Also, as $z \neq a$, the results of Case II $B$ may be applied to the effect that the pair $(a, p)$ separates $z$ and $b$. (See also the footnote below.) It will be shown that Case II D contradicts this result.

Clearly the pair $(a, p)$ separates $x_{1}$ and $b$ as well as $z$ and $b$. However, since $p$ is not a point of $L+a+b$, the component $C$ of $S-p-y_{1}$ containing $a$ must contain $b$. But as the subarc $a p$ of $T_{x}$ minus its end point $p$ is a connected set lying in $S-p-y_{1}$, it follows that the point $x_{1}$ belongs to $C$. Thus a simple arc $T$, contained in $C$, exists from $x_{1}$ to $b$. Obviously $T$ does not contain $a$, for then the subarc of $T$ from $a$ to $b$ would lie in $S-x_{1}-y_{1}$. Hence $T \subset S-a-p$, which is impossible since the pair $(a, p)$ separates $x_{1}$ and $b$. We have left then Case II C.

CASE II C. For this case consider a compact region $V$ around $p$ such that the closure $\bar{V}$ of $V$ is contained in $S-T_{y}$. Just as in Case II D the component $C_{i}$ of $S-p-y_{i}$ containing $a$ contains both $x_{i}$ and $b$, for $p$ is not a point of $L+a+b$. Thus, for every $i$ an arc $T_{i}$ exists from $x_{i}$ to $b$ and lies within $S-p-y_{i}$. As $V$ contains all the $x_{i}$ 's but a finite number let it be assumed that the $x_{i}$ 's used from now on are so chosen that $x_{i} \subset V$. Using the property that the boundary of $V, F(V)$, is closed, we see that there exists a first point $q_{i}$ of $T_{i}$, in the direction from $x_{i}$ to $b$ such that $q_{i} \subset F(V)$. Thus, the subarc $N_{i}=x_{i} q_{i}$ of $T_{i}$ lies entirely within $V$ except for its end point $q_{i}$ on $F(V)$.

Definition. The limit superior $N$ of a collection of sets $\left(N_{i}\right)$ is the set of all points $x$, such that if $R$ is any region containing $x$, $R$ contains points from an infinite number of the sets $N_{i}$. The limit inferior $M$ of the collection $\left(N_{i}\right)$ is the set of all points $y$, such that if $U$ is any region containing $y$, then $U$ contains points from all but a finite number of the sets $N_{i}$. The collection $\left(N_{i}\right)$ is said to be convergent and have limit $K=N$ if $N=M$.

* The results of Cases II A and B could also be stated: If $\sum_{1}^{\infty} x_{i}$ and $\sum_{1}^{\infty} y_{i}$ are each monotonic with limit points $p$ and $q$, respectively, where $(p+q) \cdot(a+b)=0$, then the pair $(p, q)$ separates $a$ and $b$. 
From the fact that $V$ is compact and $N_{i}$ is a continuum, the theorems on infinite collections of sets may be used to choose a sub-collection $\left(N_{v_{i}}\right)$ of $\left(N_{i}\right)$ which is convergent, whose limit $N$ is a continuum, and such that the points $x_{v_{i}}$ are monotonic on $T_{x}$.

The only point which $N$ has in common with $T_{x}$ is $p$, as is seen in the following manner. If $N \cdot a p$ contained points other than $p$, let such a point be $z$. Writing $a p=a z+z p$ and using Property VI we see that $z p$ contains all but a finite number of the points $x_{v_{i}}$. Also, if $j>k, N_{j}$ does not contain $x_{k}$, for if it did we could write $a p=a x_{j}+x_{j} p$ and then the arcs $a x_{j}$ and $T_{j}$ would contain an arc from $a$ to $b$ which would contain neither $x_{j}$ nor $y_{j}$. Hence, for $n$ so large that $x_{v_{n}} \subset z p-z$ and $S_{a_{v_{n}}}+S_{b_{v_{n}}}$, a separation of $S-x_{v_{n}}-y_{v_{n}}$, the point $z$ lies in $S_{a_{v_{n}}}$ while $\sum_{j=n+1}^{j=\infty} N_{v_{j}} \subset S_{b_{v_{n}}}$. But this is impossible since $z$ is a limit point of this latter sum.

The assumption that $N \cdot p b$ contains points other than $p$, where $p b$ is the remaining subarc of $T_{x}$, leads to a contradiction in a similar manner. Supposing that $z \subset N \cdot(p b-p)$, it is clear that every pair $\left(x_{i}, y_{i}\right)$ separates $a$ and $z$ as well as $a$ and $b$. From the note to Case IIB the pair $(p, b)$ also separates $a$ and $z$. If $S_{a}+S_{z}$ be a separation of $S-p-b$ containing $a$ and $z$ respectively, every one of the sets $\left(N_{v_{i}}-b\right)$ is contained in $S_{a}$, for $N_{i}-b$ is connected and $\sum_{1}^{\infty} x_{v_{i}} \subset S_{a}$. But this is impossible since a limit point of $\sum_{1}^{\infty} N_{v_{i}}$ is contained in $S_{z}$. Thus $N \cdot T_{x}=p$.

As $\bar{V}$ is compact and $F(V)$ is closed, the points $q_{v_{i}}$, have a limit point $q$ contained in $F(V)$. Thus, since $q \subset F(V), q \neq p$, that is, $q$ is not a point of $T_{x}$ or $T_{y}$. Let $U$ be a connected region containing $q$ such that $\bar{U} \subset S-T_{x}-T_{y}$. As $q$ is a limit point of $\sum_{1}^{\infty} q_{v_{i}}$, some $m$ exists such that $q_{v_{m}} \subset U$. Since the arc $N_{v_{m}}$ does not contain $p$ it has a last point $w$ on $a p$. By Property VI the subarc $w p$ of $a p$ contains all but a finite number of the points $x_{i}$. Choose $x_{v_{n}}$ such that $x_{v_{n}} \subset w p-w$ and $n>m$. Since the $x_{v_{i}}$ 's are monotonic, the subarc $a x_{v_{m}}$ of $T_{x}$ is contained within $S-x_{v_{n}}-y_{v_{n}}$ as are also $N_{v_{m}}$ and $U$. From the preceding paragraph $N \subset S-x_{i}-y_{i}$. Likewise, the subarc $p b$ of $T_{x}$ is also contained in $S-x_{i}-y_{i}$. Hence, $G=a x_{v_{m}}+N_{v_{m}}+U+N+p b$ lies within $S-x_{v_{n}}-y_{v_{n}}$. But this is impossible since $G$ is a connected set containing both $a$ and $b$ while the pair $\left(x_{v_{n}}, y_{v_{n}}\right)$ separates $a$ and $b$.

Thus the theorem is established that $L+a+b$ is closed and compact. The assumption need not be made that $S$ has no cut 
point in general but merely that no single point $x$ separates $a$ and $b$. Under this latter assumption the $\operatorname{arcs} T_{x}$ and $T_{y}$ exist.

\section{PART II}

The second part of this paper treats the following theorem:

If $G$ is any collection of closed, mutually exclusive and nonseparated sets $X$ separating any two fixed points $a$ and $b$ of a connected and locally connected, separable metric space $S$, then the elements of $G$ are ordered.* Further, any infinite monotonic subcollection $\left(X_{i}\right)$ of $G$ is convergent and has a non-vacuous limit $M$ which separates $a$ and $b$ if $M \subset S-a-b$.

Definition. By non-separated is meant that if $X_{i}$ and $X_{j}$ are any two elements of $G$ and $S_{a_{i}}+S_{b_{i}}$ is.a separation of $S-X_{i}$, the set $X_{j}$ is contained entirely within $S_{a_{i}}$ or $S_{b_{i}}$.

The ordering of $G$ is defined as follows: $X_{i}$ is said to precede $X_{j}, X_{i} \propto X_{j}$, if $X_{j} \subset S_{b_{i}}$. Some consequences of this definition are: either $X_{i} \propto X_{j}$ or $X_{j} \propto X_{i}$; if $X_{i} \propto X_{j}, X_{j}$ does not precede $X_{i}$; if $X_{i} \propto X_{j} \propto X_{k}$, then $X_{i} \propto X_{k}$.

Suppose that $\left(X_{i}\right)$ is any infinite monotonic plus collection of sets $X_{i}$, that is, if $S_{a_{i}}+S_{b_{i}}$ is a separation of $S-X_{i}$ then $\sum_{k=1}^{i-1} X_{k}$ $\subset S_{a_{i}}$ while $\sum_{k=i+1}^{\infty} X_{k} \subset S_{b_{i}}$. From this it is easily seen that no point of the limit superior of $\left(X_{i}\right)$ is contained in any $S_{a_{i}}$ or $X_{i}$, for that point would then be a limit point of $S_{b_{i}}$. It will be shown first that the limit superior $X$ of $\left(X_{i}\right)$ is non-vacuous. If $S_{a}=\sum_{1}^{\infty} S_{a_{i}}$ and $S_{b}=\prod_{1}^{\infty} S_{b_{i}}$, it is easily seen that $S_{a} \cdot S_{b}=0$, for otherwise some $i$ would exist such that $S_{a_{i}} \cdot S_{b_{i}}$ would not be vacuous. Now $S=S_{a}+S_{b}$, for if $z$ is a point of $S$, either $z$ is a point of some $X_{i} \subset S_{a_{i}+1} \subset S_{a}$ or not. If not, either $z$ is contained in every $S_{b_{i}}$, that is, $z \subset S_{b}$, or, since $z$ is not contained in $\sum_{1}^{\infty} X_{i}$, some $n$ exists such that $z \subset S_{a_{n}} \subset S_{a}$. Now $S_{a_{i}}$ is an open set, for if a point $p \subset S_{a_{i}}$, since $X_{i}$ is closed, a connected region $R$ exists such that $p \subset R \subset S-X_{i}$. That is, $R \subset S_{a_{i}}$, and hence, since the sum of any number of open sets is again an open set, $S_{a}$ is open. On the assumption that $\lim \sup \left(X_{i}\right)=X=0$, no point of $S_{b}$ is a limit point of $\sum_{1}^{\infty} X_{i}$. Thus, if $p$ is a point of $S_{b}$, a connected region $R$ exists such that $p \subset R \subset S-\sum_{1}^{\infty} X_{i}$. As $p$ is contained in every $S_{b_{i}}$, it follows that $R$ is also. Therefore, $S_{a}$ and $S_{b}$ are

* For references on the ordering of the elements of $G$ see G. T. Whyburn, Non-separated cuttings of connected point sets, Transactions of this Society, vol. 33 (1931). 
mutually exclusive open sets containing $a$ and $b$ respectively. But two mutually exclusive open sets are mutually separated, so that the assumption that $X=0$ leads to the contradiction that $S$ is not connected.

The supposition that $\left(X_{i}\right)$ is not convergent again leads to a contradiction. For if $\left(X_{i}\right)$ is not convergent, an infinite subcollection $\left(X_{n_{i}}\right)$ of the $X_{i}$ 's exists such that $\lim \sup \left(X_{i}\right)=X$ $\neq \lim \sup \left(X_{n_{i}}\right)=N$. Choose the $X_{n_{i}}$ 's such that they are monotonic and form $S_{a}=\sum_{1}^{\infty} S_{a_{n i}}$ and $S_{b}=\prod_{1}^{\infty} S_{b_{n i}}-N$. Just as before $S_{a}$ and $S_{b}$ are mutually exclusive open sets whose sum is $S-N$. However, this is impossible since $X-N \neq 0$ and is contained in $S_{b}$ while $\sum_{1}^{\infty} X_{i} \subset S_{a}$ (given any $X_{i}$ an $X_{n_{i}}$ exists such that $X_{i} \propto X_{n_{i}}$, that is, $\left.X_{i} \subset S_{a_{n i}} \subset S_{a}\right)$. Thus we see that the collection $\left(X_{i}\right)$ is convergent.

Since every monotonic collection is either monotonic plus or monotonic minus, an interchange of $a$ and $b$ will take care of the negative case. It merely remains to show that the limit $M$ of $\left(X_{i}\right)$ separates $a$ and $b$ if $M \subset S-a-b$. Assuming that the collection $\left(X_{i}\right)$ is monotonic plus, and forming as before $S_{a}=\sum_{1}^{\infty} S_{a_{i}}$ and $S_{b}=\prod_{1}^{\infty} S_{b_{i}}-M$, we see that the sets $S_{a}$ and $S_{b}$, being mutually exclusive open sets whose sum is $S-M$, form a separation of $S-M$. Also, as neither $a$ nor $b$ was contained in $M, a \subset S_{a}$ and $b \subset S_{b}$.

The Johns Hopkins University 